\title{
MHD on Convective Mass Transfer in Annular Flow: Catheter Based Drug Release
}

\author{
Punith Kumar Deshapande ${ }^{1(\mathbb{D})}$, Indira Rama Rao ${ }^{2} \mathbb{( D )}$, Sravan Kumar Thavada ${ }^{1, *(\mathbb{D})}$ \\ 1 Department of Mathematics, SoAS, REVA University, Bangalore-560064, Karnataka, India; punithkumardn@ reva.edu.in \\ (P.K.D.); thavadasravankumar@gmail.com (S.K.T.); \\ 2 Department of Mathematics, Nitte Meenakshi Institute of Technology, Bangalore-560064, Karnataka, India; \\ indiradiv@gmail.com (I.R.R.); \\ * Correspondence: thavadasravankumar@gmail.com (S.K.T.);
}

Scopus Author ID 56872884700

Received: 13.06.2021; Revised: 10.09.2021; Accepted: 14.09.2021; Published: 19.11.2021

\begin{abstract}
In the present study, convective diffusive mass transfer is considered, along with effects of particle drag under the influence of a magnetic field concerning drug delivery in the presence of the catheter. A concentric annular region is created by the presence of a catheter, and the effects of which on mass transfer are considered. A model on the hydrodynamics of the fluid, blood flow, and convective diffusive mass transfer of the species is presented. Here, an attempt is made to analyze a drug delivery method for delivering a drug to a specific site in the body and for this analysis, considered a channel bounded by the tissue region where the drug is targeted. The magnetic field induces pulsatile flow, which affects the mass transfer. The graphs predict that the mass transfer increases from the lumen region to the tissue region. Peclet number and magnetic parameter are the parameters that significantly affect carrying drugs towards the tissue. The results are well agreed with the physical phenomena of the problem as well as many biomedical applications.
\end{abstract}

Keywords: generalized dispersion model; Newtonian fluid; magnetic field; dispersion coefficient; interphase mass transfer.

(C) 2021 by the authors. This article is an open-access article distributed under the terms and conditions of the Creative Commons Attribution (CC BY) license (https://creativecommons.org/licenses/by/4.0/).

\section{Introduction}

A catheter is a tube that can be inserted into a body cavity, duct, or vessel. It is a thin, flexible tube. A catheter left inside the body either temporarily or permanently is referred to as an indwelling catheter. A catheter can be used for different medicinal applications. It is used to drain urine from the kidney, angioplasty, angiography, measuring blood pressure, etc. It can also be used for the administration of intravenous fluids, medication, or providing nutrition.

The study of convective diffusive mass transfer has an important role in understanding the administration of drugs, transport of LDL, which is the cause of Atherosclerosis, transport of oxygen and nutrients, etc. The intravenous fluid injected gets dispersed and is carried to the tissue region surrounding it. The dispersion of mass in blood vessels is influenced by conductive blood vessel walls examined by Sarkar and Jayaraman et al. [1]. Shankarasubramanian and Gill [2] have analyzed solute dispersion through a circular tube, developing a generalized dispersion model. Mazumdar and Das [3] have studied the effect of wall conductance on the axial dispersion in the pulsatile tube flow.

Jiang and Grotberg [4] studied the dispersion of bolus contaminant in a straight tube with the oscillatory flow and weak conductive walls. Balasubramanian et al. [5] have studied 
the combined effect of secondary flows and shown that secondary flow has a negligible effect. Pedley and Kamm [6] studied axial mass transport in an annular region by asymptotic analysis to limit a small annular gap. Srivastava et al. [7] has studied the particle fluid suspension model of blood flow through a stenotic blood vessel. Recently, Umadevi et al. [8] have studied the effect of particle drag using the Gill and Shankarasubramanian model. A similar analysis is done in studying the behavior of dispersion in the case of oscillatory flows in uniform conduits. Also, it is shown that diffusion of heat and other diffusible properties through the interior of the fluid by turbulent motion [9-11]. Pedram and Ehasan [12] have been developed to study nanofluid transportation in bio tissue in the case of magnetic hyperthermia. Kumar et al. [13] studied the magnetic targeting of the drug numerically under the effect of the applied magnetic field. Shah et al. [14] considered an innovative model that has been developed to study the electro-osmotic flow of Couette nanofluids. Kumaresan et al. [15] studied the effect of stenosis on magneto nanoparticles distributions in the presence of the magnetic field. Nadjia and Nadji [16] have examined the heat transfer characteristics of various parameters of ferrofluids. Shah et al. [17] used an implicit method (Keller Box) to analyze the nanofluid flow in the case of stretching sheets considering the heat transfer through convection. They concluded that heat transfer varies for different classes of nanofluids. Ibrahim and Gadisa [18] examined the heat transfer rate decreases when the nanoparticle volume fraction increases for cu-water. Naveed Khan et al. [19] considered nanofluid flow in the case of the vertical plate to study the heat generation effects due to natural convection. They proved that as the volume concentration increases, the temperature and heat transfer rate also increases. Meanwhile, Sulochana et al. [20] and Rafique et al. [21] worked on the free convection flow of nanofluids in the case of the vertical plate of infinite length and studied the effects of magnetic field on it. The solution to the problem is obtained using a numerical method.

Many researchers have done extensive work on new manufacturing methods, and new models for non-Newtonian fluids are proposed [22-24]. It is observed that the application of boundary layer theory for the flows is relevant for the flow analysis through annulus in the engineering domain. They are applied in biological fluid movement, manufacturing of glass fiber, and wire drawing.

In the present study, convective diffusive mass transfer is considered, along with the effects of particle drag under the influence of a magnetic field with reference to drug delivery in the presence of a catheter. A concentric annular region is created by the presence of a catheter and the effects on mass transfer.

\section{Mathematical Formulation}

Consider the physical configuration of the catheterized artery shown in Figure 1. In this diagram, we consider the concentric annular geometry with $R_{0}$ as the radius of the artery representing the outer tube and $K R_{0}$ is the radius of the catheter representing the inner tube $(K<1)$. Let us consider the axi symmetric flow in the concentric annulus where the following fluid is a dusty viscous fluid.

We know that diffusive and advective processes govern the mass transport through the artery wall. The most important aspect of drug transport through the wall is the metabolic consumption of drugs by the wall tissue. To avoid the decay of the drug, we apply a first-order reaction and incorporate the external magnetic effect. 


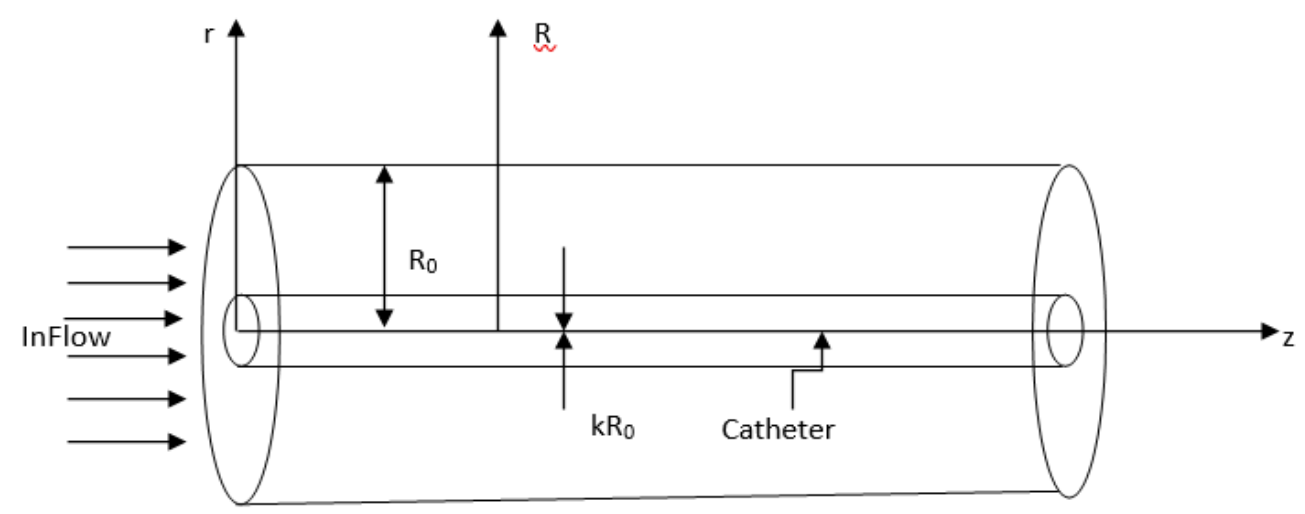

Figure 1. The physical configuration of the catheterized artery.

The governing equations in cylindrical coordinates $(R, \theta, Z)$ for the above-explained configuration are given by

$$
\begin{aligned}
& \rho \frac{\partial w}{\partial t}=-\frac{\partial P}{\partial z}+\mu\left[\frac{\partial^{2} w}{\partial r^{2}}+\frac{1}{r} \frac{\partial w}{\partial r}\right]+K N(v-w)-\sigma_{0} B^{2} w \\
& N m \frac{\partial v}{\partial t}=K N(w-v)
\end{aligned}
$$

Where $\mu$ is the viscosity of the fluid is, $m$ is the Brinkman viscosity of the fluid, $v$ is the velocity of the dusty particle, $N$ is the number density of the particle, $K=3 \mu \pi d$ is the stokes drag term, $d$ is the diameter of the dusty particle, $N m$ is the mass of the dusty particle, $\sigma_{0}$ is the stress tensor, $B^{2}$ is the magnetic effect on the flow, $w$ is the velocity of the fluid phase, $P$ is the pressure, and $\rho$ is the density of the fluid.

To solve (1) and (2) the no-slip boundary conditions are

$w=0 \quad$ at $\quad R=k R_{0}$ and $R=R_{0}$

Assuming $r=\frac{R}{R_{0}}, w=W(r) e^{-n t}, v=V(r) e^{-n t}, \frac{\partial P}{\partial z}=-P e^{-n t}$ and eliminating $v$ from (2), we get

$\frac{d^{2} W}{d r^{2}}+\frac{1}{r} \frac{d W}{d r}+\lambda^{2} W=P$

Where $P=-\frac{p}{\mu}, \lambda^{2}=\left[\frac{K N}{\rho} \frac{n \Gamma}{1-n \Gamma}-\frac{\sigma_{0} B^{2}}{\mu}+\frac{\rho n}{\mu}\right], \Gamma=\frac{m}{k}, \lambda$ is the drag parameter, $\Gamma$ is the relaxation time, $\rho$ is the pressure gradient, and $W$ is the non-dimensional velocity of the fluid phase.

Equation (3) in non-dimensional form is

$W=0 \quad$ at $\quad r=K$

$\frac{\partial W}{\partial r}=-\alpha W$ at $r=1$

The solution of (4) using (5) and (6) is

$W(r)=A J_{0}(\lambda r)+B Y_{0}(\lambda r)$

Where $A=\frac{P}{\lambda^{2}}\left[\frac{\alpha\left(Y_{0}(\lambda k)-Y_{0}(\lambda)\right)-\lambda Y_{0}{ }^{\prime}(\lambda)}{\alpha\left\{\left(J_{0}(\lambda) Y_{0}(\lambda k)\right)-Y_{0}(\lambda) J_{0}(\lambda k)\right\}+\lambda\left\{\left(J_{0}{ }^{\prime}(\lambda) Y_{0}(\lambda k)\right)-Y_{0}{ }^{\prime}(\lambda) J_{0}(\lambda k)\right\}}\right]$ 


$$
B=\frac{P}{\lambda^{2}}\left[\frac{\alpha\left\{J_{0}(\lambda)-J_{0}(\lambda k)\right\}+\lambda J_{0}{ }^{\prime}(\lambda)}{\alpha\left\{\left(J_{0}(\lambda) Y_{0}(\lambda k)\right)-Y_{0}(\lambda) J_{0}(\lambda k)\right\}+\lambda\left\{\left(J_{0}{ }^{\prime}(\lambda) Y_{0}(\lambda k)\right)-Y_{0}{ }^{\prime}(\lambda) J_{0}(\lambda k)\right\}}\right]
$$

\section{Dispersion Model}

To develop a convective-diffusion model for the physical configuration under study, we consider the convective-diffusion equation in the cylindrical coordinate system $(R, \theta, Z)$ given by

$\frac{\partial c}{\partial t}+w(t, R) \frac{\partial c}{\partial z}=D_{m}\left\{\left(\frac{\partial^{2} c}{\partial R^{2}}+\frac{1}{R} \frac{\partial c}{\partial R}\right)+\frac{\partial^{2} c}{\partial z^{2}}\right\}$

Where $t$ is the time, $c$ is the concentration of the solute, $R$ is the radial coordinate, $z$ is the axial coordinate, and $w$ is the axial velocity.

To solve (9), we define initial and boundary conditions as:

$c(0, R, \theta, z)=c_{0} \psi_{2}(z) \zeta_{1}(R)$,

where $\psi_{2}(z)$ is a function of $z$,

$$
\begin{aligned}
& \zeta_{1}(R)=\left\{\begin{array}{rl}
R_{0}, & k R_{0}<R \leq a R_{0} \\
0, & a R_{0}<R \leq R_{0}
\end{array} .\right. \\
& \frac{\partial \mathrm{c}}{\partial \mathrm{R}}=-\mathrm{kc} \quad \text { at } \quad \mathrm{R}=R_{0} \\
& \frac{\partial \mathrm{c}}{\partial \mathrm{R}}=0 \quad \text { at } \quad \mathrm{R}=k R_{0}
\end{aligned}
$$

Non-dimensionalising (9) to (12b) using the following

$$
\begin{aligned}
& \mathrm{c}^{*}=\frac{\mathrm{c}}{\mathrm{c}_{0}}, \mathrm{z}^{*}=\frac{\mathrm{z}}{\mathrm{D}_{\mathrm{m}} / \mathrm{R}_{0}{ }^{2} \mathrm{w}_{0}}, \mathrm{r}^{*}=\frac{\mathrm{r}}{\mathrm{R}_{0}}, \mathrm{w}^{*}=\frac{\mathrm{w}}{\mathrm{W}_{0}}, \mathrm{t}^{*}=\frac{\mathrm{t}}{\mathrm{R}_{0}{ }^{2} / \mathrm{D}_{\mathrm{m}}}, \mathrm{P}_{\mathrm{e}}=\frac{\mathrm{R}_{0} \mathrm{w}_{0}}{\mathrm{D}_{\mathrm{m}}}, \text { we get } \\
& \frac{\partial c}{\partial \tau}+w(r, \tau) \frac{\partial c}{\partial z}=\frac{\partial^{2} c}{\partial r^{2}}+\frac{1}{r} \frac{\partial c}{\partial r}+\frac{1}{\mathrm{P}_{\mathrm{e}}{ }^{2}} \frac{\partial^{2} c}{\partial z^{2}} \\
& c(0, r, \theta, z)=B_{2}(z) B_{1}(r), \\
& \frac{\partial \mathrm{c}}{\partial \mathrm{r}}=-\beta \mathrm{c} \quad \text { at } \quad r=1 \\
& \frac{\partial \mathrm{c}}{\partial \mathrm{r}}=0 \quad \text { at } \quad r=k
\end{aligned}
$$

where $B_{1}(r)=\left\{\begin{array}{ll}1, & k<r \leq a \\ 0, & a<r \leq 1\end{array}, B_{2}(z)=\frac{\delta(z)}{a^{2} P_{e}}, \tau\right.$ is the non-dimensional time, $\mathrm{c}_{0}$ is the reference concentration, $\mathrm{D}_{\mathrm{m}}$ is the molecular diffusivity, $\mathrm{W}_{0}$ is the mean axial velocity, $\mathrm{R}_{0}$ is the radius of the tube, $\mathrm{P}_{\mathrm{e}}$ is the Peclet number, $w(r, \tau)$ is the non-dimensional axial velocity, $\beta$ is the non-dimensional wall reaction parameter.

By using the generalized dispersion model proposed by Gill and Sankarasubramanian (1970) and solving (13) using (13a) to (13c), we get

$\mathrm{c}(\tau, \mathrm{r}, \mathrm{z})=\sum_{\mathrm{n}=0}^{\infty} \mathrm{f}_{\mathrm{n}}(\tau, \mathrm{r}) \frac{\partial^{\mathrm{n}} \mathrm{c}_{\mathrm{m}}}{\partial \mathrm{z}^{\mathrm{n}}}$

where the mean concentration ' $\mathrm{c}_{\mathrm{m}}$ ' is expressed as 
$c_{m}=\frac{\int_{0}^{2 \pi} \int_{k}^{1} r c d r d \theta}{\int_{0}^{2 \pi} \int_{k}^{1} r d r d \theta}=\frac{2}{\left(1-k^{2}\right)} \int_{k}^{1} r c d r$

Equation (13) using (14) takes the form

$\frac{\partial c_{m}}{\partial \tau}=\frac{1}{P_{e}^{2}} \frac{\partial^{2} c_{m}}{\partial r^{2}}+\left|\frac{\partial c_{m}}{\partial r}\right|_{k}^{1}-\frac{\partial}{\partial r} \int_{k}^{1} U c d r$

Let us introduce the dispersion coefficient in the model as

$\frac{\partial c_{m}}{\partial \tau}=\sum_{\mathrm{i}=0}^{\infty} \mathrm{M}_{i}(\tau) \frac{\partial^{\mathrm{i}} \mathrm{C}_{\mathrm{m}}}{\partial \mathrm{r}^{\mathrm{i}}}$

The values of $M_{i}(\tau)^{\prime} s$ can be obtained from the following result:

$M_{i}(\tau)=\frac{2}{\left(1-k^{2}\right)} \frac{\partial}{\partial r} f_{i}\left(\tau_{i}\right)+\frac{\delta_{i, 2}}{P e^{2}}-\frac{2}{\left(1-k^{2}\right)} \int_{k}^{1} r w(\tau, r) f_{i-1} d r \quad i=0,1,2, \ldots \ldots$,

Where $f_{-1}=f_{-2}=0, \delta_{i, 2}$ is the kronecker delta, $\frac{2}{\left(1-k^{2}\right)} \frac{\partial}{\partial r} f_{i}\left(\tau_{i}\right)$ is due to the first-order heterogeneous reaction at the outer wall of the tube.

Truncating higher terms in (17), we get

$\frac{\partial c_{m}}{\partial \tau}=M_{0}(\tau) c_{m}+M_{1}(\tau) \frac{\partial c_{m}}{\partial z}+M_{2}(\tau) \frac{\partial^{2} c_{m}}{\partial z^{2}}$

Where $M_{0}(\tau)$ is the absorption parameter, $M_{1}(\tau)$ is the velocity of the reactive tracer and $M_{2}(\tau)$ is a modification in the convective dispersion because of absorption. $M_{1}(\tau)$ and $M_{2}(\tau)$ are convective and dispersion coefficients respectively. The values of $M_{i}(\tau)$ and $f_{k}(k=$ $1,2,3, .$.$) are required to solve (19).$

Substituting (14) in (13) and using (19), we obtain a set of differential equations for $f_{k}$ as shown below:

$\frac{\partial f_{k}}{\partial \tau}=\frac{\partial^{2} f_{k}}{\partial r^{2}}+\frac{1}{r} \frac{\partial f_{k}}{\partial r}-w(\tau, r) f_{k-1}+\frac{1}{P e^{2}} f_{k-2}+\sum_{n=0}^{\infty} f_{n-i} M_{i} \quad k=0,1,2, \ldots$

To find $c_{m}$ and $f_{k}$ the required initial and boundary conditions are

$c_{m}(0, z)=\frac{2}{\left(1-k^{2}\right)} \int_{k}^{1} r B_{1}(r) d r$

$c(0, r, z)=f_{0}(0, r) c_{m}(0, z)$

$f_{k}(0, r)=0 \quad$ for $\quad k=1,2,3, \ldots \ldots$

$f_{k}(0, r)=\left\{\begin{array}{cc}\frac{\left(1-k^{2}\right)}{2} \frac{B_{1}(r)}{\int_{k}^{1} r B_{1}(r) d r}, & k=0 \\ 0, & k \neq 0\end{array}\right.$

$\frac{\partial f_{k}}{\partial r}(\tau, 1)=-\beta f_{k}(\tau, 1)$

$\frac{\partial f_{k}}{\partial r}(r, n)=0$, for $k=0,1,2,3, \ldots$

Using (15) into (14), we get

$\int_{k}^{1} r f_{n}(\tau, r) d r=\frac{\left(1-k^{2}\right)}{2} \delta_{n, 0}$ 
Since the equations are coupled to find $M_{2}(\tau)$, we need to find $\left(f_{n}, M_{n}\right)$ for $n=0,1,2$ in pairs. The functions $f_{0}$ and exchange coefficient $M_{0}$ are independent of velocity field and can be solved directly.

From (18), we get

$M_{0}(\tau)=\frac{2}{\left(1-k^{2}\right)} \frac{\partial f_{0}}{\partial r}(\tau, 1)$

The coefficient $M_{0}(\tau)$ is decoupled with the equation obtained from (20) given by

$\frac{\partial f_{0}}{\partial \tau}=\frac{\partial^{2} f_{0}}{\partial r^{2}}+\frac{1}{r} \frac{\partial f_{0}}{\partial r}-M_{0} f_{0}$

The solution of (24) may be formulated as

$f_{0}(\tau, r)=e^{-\int_{0}^{\tau} M_{0}(\eta) d \eta} g_{0}(\tau, r)$

Where $g_{0}(\tau, r)$ must satisfy $\frac{\partial g_{0}}{\partial \tau}=\frac{\partial^{2} g_{0}}{\partial r^{2}}+\frac{1}{r} \frac{\partial g_{0}}{\partial r}$

With boundary conditions

$g_{0}(0, r)=\frac{\left(1-k^{2}\right)}{2} \frac{B_{1}(r)}{\int_{k}^{1} r B_{1}(r) d r}$,

$\frac{\partial g_{0}}{\partial r}(\tau, 1)=-\beta g_{0}(\tau, 1)$,

$\frac{\partial g_{0}}{\partial r}(\tau, k)=0$

The solution of (26a) using (26b) to (26d) is

$g_{0}(\tau, r)=\sum_{n=0}^{\infty} \frac{A_{n}}{J_{1}\left(\mu_{n} k\right)} E_{n}\left(\mu_{n} r\right) e^{-\mu_{0}^{2} \tau}$

Where $A_{n}=\frac{\mu_{n}^{2}\left(1-k^{2}\right) J_{1}\left(\mu_{n} k\right) \int_{k}^{1} r B_{1}(r) E_{n}\left(\mu_{n} r\right) d r}{\left(\left(\mu_{n}^{2}+\beta^{2}\right)\left\{E_{n}\left(\mu_{n}\right)\right\}^{2}-k^{2} \mu_{n}^{2}\left\{E_{n}\left(\mu_{n} k\right)\right\}^{2}\right) \int_{k}^{1} r B_{1}(r) d r}$

$$
E_{n}\left(\mu_{n} r\right)=Y_{0}\left(\mu_{n} r\right) J_{1}\left(\mu_{n} k\right)-Y_{1}\left(\mu_{n} k\right) J_{0}\left(\mu_{n} r\right)
$$

And $\mu_{n}$ 's are eigenvalues satisfying the following equation:

$\mu_{n}\left(Y_{1}\left(\mu_{n} k\right) J_{1}\left(\mu_{n}\right)-J_{1}\left(\mu_{n} k\right) Y_{1}\left(\mu_{n}\right)\right)+\beta\left(Y_{0}\left(\mu_{n}\right) J_{1}\left(\mu_{n} k\right)-Y_{1}\left(\mu_{n} k\right) J_{0}\left(\mu_{n}\right)\right)=0$

In the above equation $J_{0}, Y_{0}$ are Bessel functions of order ZERO and $J_{1}, Y_{1}$ are Bessel functions of order ONE.

From (22), we have

$\int_{k}^{1} r f_{0}(\tau, r) d r=\frac{\left(1-k^{2}\right)}{2}$

Using (25) in (28), we get

$e^{-\int_{0}^{r} M_{0}(\tau) d \tau}=\frac{\left(1-k^{2}\right)}{2} \frac{1}{\int_{k}^{1} r g_{0}(\tau, r) d r}$

From (25) and (29), we obtain $f_{0}(\tau, r)$ as

$f_{0}(\tau, r)=\frac{\left(1-k^{2}\right)}{2} \frac{g_{0}(\tau, r)}{\int_{k}^{1} r g_{0}(\tau, r) d r}$

From (23) and (30), we obtain 
$M_{0}(\tau)=-\frac{\sum_{n=0}^{\infty} \frac{A_{n}}{J_{1}\left(\mu_{n} k\right)} \mu_{n}\left[Y_{1}\left(\mu_{n}\right) J_{1}\left(\mu_{n} k\right)-J_{1}\left(\mu_{n}\right) Y_{1}\left(\mu_{n} k\right)\right] e^{-\mu_{n}^{2} \tau}}{\sum_{n=0}^{\infty} \frac{A_{n}}{J_{1}\left(\mu_{n} k\right)}\left[Y_{1}\left(\mu_{n}\right) J_{1}\left(\mu_{n} k\right)-J_{1}\left(\mu_{n}\right) Y_{1}\left(\mu_{n} k\right)\right] e^{-\mu_{n}^{2} \tau}}$

During the process of finding the remaining functions $\left(f_{n}, M_{n}\right)$ for $n=1,2,3, \ldots$ the computation becomes complex. Hence we consider larger time solutions w.r.t steady flow conditions. By this consideration, the defined model represents the asymptotic results under steady flow conditions.

As $\tau \rightarrow \infty$, Equations (30) and (31) give the following asymptotic representations for $f_{0}$ and $M_{0}$.

$f_{0}(\infty, r)=\frac{\left(1-k^{2}\right)}{2} \frac{\mu_{0}\left[Y_{0}\left(\mu_{0} r\right) J_{1}\left(\mu_{0} k\right)-J_{0}\left(\mu_{0} r\right) Y_{1}\left(\mu_{0} k\right)\right]}{\left[Y_{1}\left(\mu_{0}\right) J_{1}\left(\mu_{0} k\right)-J_{1}\left(\mu_{0}\right) Y_{1}\left(\mu_{0} k\right)\right]}$

$M_{0}(\infty)=-\mu_{0}^{2}$,

where $\mu_{0}$ is the first (lowest in magnitude) root of equation (27d)

From (20), we have $f_{1}(r)$ satisfying the following equation

$\frac{\partial^{2} f_{1}}{\partial r^{2}}+\frac{1}{r} \frac{\partial f_{1}}{\partial r}+\mu_{0} f_{1}=w(r) f_{0}+M_{1} f_{0}$.

With boundary conditions

$\frac{\partial f_{1}}{\partial r}(1)=-\beta f_{1}(1)$

$\frac{\partial f_{1}}{\partial r}(k)=0$,

From (18) and (35a) we have

$M_{1}=-\frac{2}{\left(1-k^{2}\right)}\left[\beta f_{1}(1)+\int_{k}^{1} r w(r) f_{0} d r\right]$

Equations (34) and (36) are coupled, and equation (36) is not known explicitly as $f_{1}(1)$ is unknown at this stage. By multiplying equation (34) with $r E_{0}\left(\mu_{0} r\right)$, and integrating from $\mathrm{k}$ to 1 with respect to $\mathrm{r}$, it is observed that the left-hand side becomes zero leaving the nonzero right-hand side as

$M_{1}(\tau)=-\frac{\int_{k}^{1} r w(r) E_{0}\left(\mu_{0} r\right) f_{0}(r) d r}{\int_{k}^{1} r E_{0}\left(\mu_{0} r\right) f_{0}(r) d r}$ giving

$M_{1}(\tau)=\frac{-4 \mu_{0}\left[Y_{1}\left(\mu_{0}\right) J_{1}\left(\mu_{0} k\right)-J_{1}\left(\mu_{0}\right) Y_{1}\left(\mu_{0} k\right)\right] \int_{k}^{1} r w(r) E_{0}\left(\mu_{0} r\right) f_{0} d r}{\left(1-k^{2}\right)\left[\left(\mu_{0}^{2}+\beta^{2}\right)\left\{E_{0}\left(\mu_{0}\right)\right\}^{2}-k^{2} \mu_{0}^{2}\left\{E_{0}\left(\mu_{0} k\right)\right\}^{2}\right]}$

Using (34) and (37), we get $f_{1}$ satisfying the boundary conditions (35a) and (35b) as

$f_{1}(r)=\sum_{n=0}^{\infty} \frac{A_{1 n} E_{n}\left(\mu_{n} r\right)}{J_{1}\left(\mu_{n} k\right)}$

where

$A_{1 n}=\left\{\begin{array}{cl}\frac{\int_{k}^{1} r\left[w(r)+M_{0}\right] f_{0}(r) E_{n}\left(\mu_{n} r\right) d r}{J_{1}\left(\mu_{n} k\right)\left(\mu_{n}^{2}-\mu_{0}^{2}\right)}, & \text { for } n \geq 1 \\ -\frac{J_{1}\left(\mu_{0} k\right)}{\int_{k}^{1} r E_{0}\left(\mu_{0} r\right) f_{0} d r} \sum_{n=1}^{\infty} \frac{A_{1 n}}{J_{1}\left(\mu_{n} k\right)} \int_{k}^{1} r E_{n}\left(\mu_{n} r\right) d r, & \text { for } n=0\end{array}\right.$

For $=0$, the expression for $A_{1 n}$ is obtained from equation (22), i.e $\int_{k}^{1} r f_{1} d r=0$. 
By applying the same procedure used to find $M_{1}(\tau)$, we obtain $M_{2}(\tau)$ as

$M_{2}(\tau)=\frac{1}{P e^{2}}+\frac{-\int_{k}^{1} r\left[w(r)+M_{1}\right] f_{1}(r) E_{0}\left(\mu_{0} r\right) d r}{\int_{k}^{1} r E_{0}\left(\mu_{0} r\right) f_{0}(r) d r}$

\section{Results and Discussion}

A catheter is used for different purposes. The presence of a catheter causes variations in the presence and amount of blood flowing inside, affecting the solute transfer process. The absorption by a wall is enhanced by the presence of a magnetic field which causes more solute to get convected to the wall. Wall absorption parameter $\beta$ affects the exchange coefficient, asymptotic convection, and diffusion coefficients.

Figures 2-4 show the plot of a radial velocity profile for different catheter radius magnetic parameters $M$ and permeability $\alpha$. Increases in catheter radius increase the velocity. This is due to the fact that the area of the cross-section decreases with an increase in catheter radius. But it is necessary to maintain the same amount of fluid flow to facilitate tissue needs around. Hence velocity increases.

As the permeability increases, velocity near the center of the annular region (created by the catheter) increases but closer to the wall of the tissue region (where the drug is targeted), velocity reduces. This is due to the loss of fluid at a permeable wall. The increase in magnetic field causes a pulsatile flow which can be seen in Figure 3. Figures 5 and 6 show convection coefficient versus absorption parameters for different magnetic drag and different permeability. As magnetic field increases $-M_{1}$ increases showing more solute gets convected with the fluid, thereby decreasing absorption at the wall. As absorption increases $-M_{1}$ decreases showing convection coefficient affects inversely to the solute of absorption.

As permeability increases, convection decreases due to the loss of fluid. Hence increase in the permeability parameter results in a decrease in convection coefficient. Figures 5-6 show a plot of diffusion coefficient versus absorption parameter $\beta$ for different values of a magnetic parameter, permeability, and Peclet number. Diffusion increases with increasing absorption parameters. The diffusion coefficient increases with increased permeability due to enhancement in fluid convection of fluid towards the wall, thereby increasing absorption.

Increase in magnetic field decreases $M_{2}$ due to the fact that the magnetic field increases the velocity of the fluid, thereby increasing the convection. An increase in Peclet number shows a decrease in diffusion hence decrease in $M_{2}$. Peclet number and magnetic field influence diffusion significantly. The effect of an increase in a magnetic field is to create pulsatile motion.

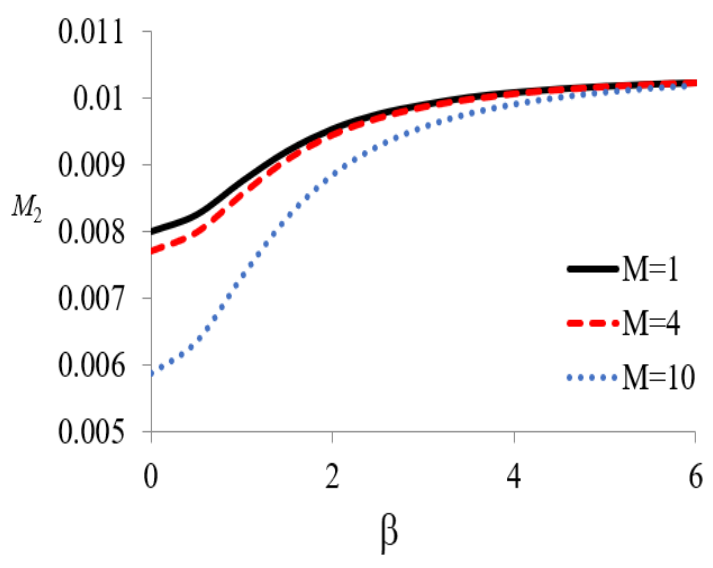

https://biointerfaceresearch.com/

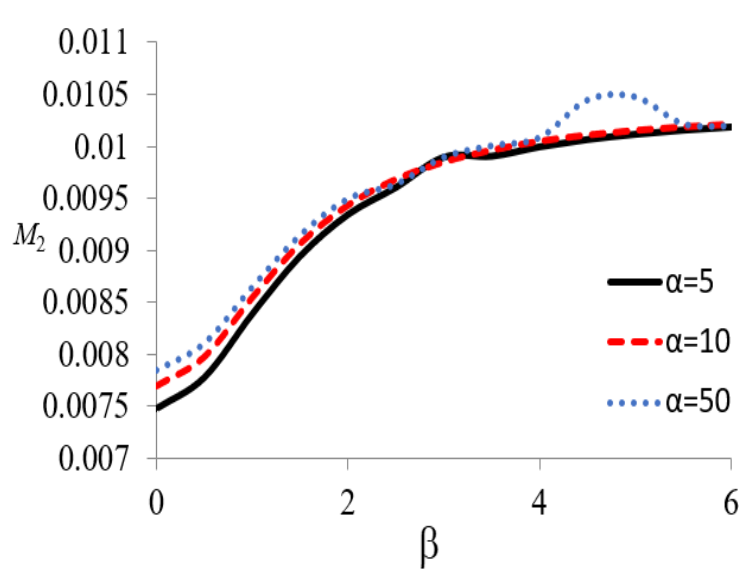


Figure 2. the plot of asymptotic dispersion coefficient vs. absorption parameter for different values of magnetic drag.

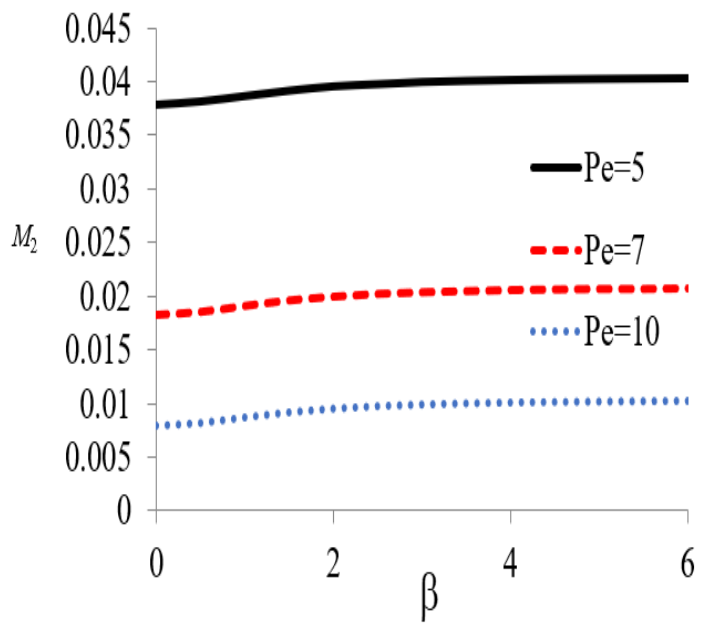

Figure 4. The plot of asymptotic dispersion coefficient vs. absorption parameter for different values of peclet number.
Figure 3. The plot of asymptotic dispersion coefficient vs. absorption parameter for different values of permeability.

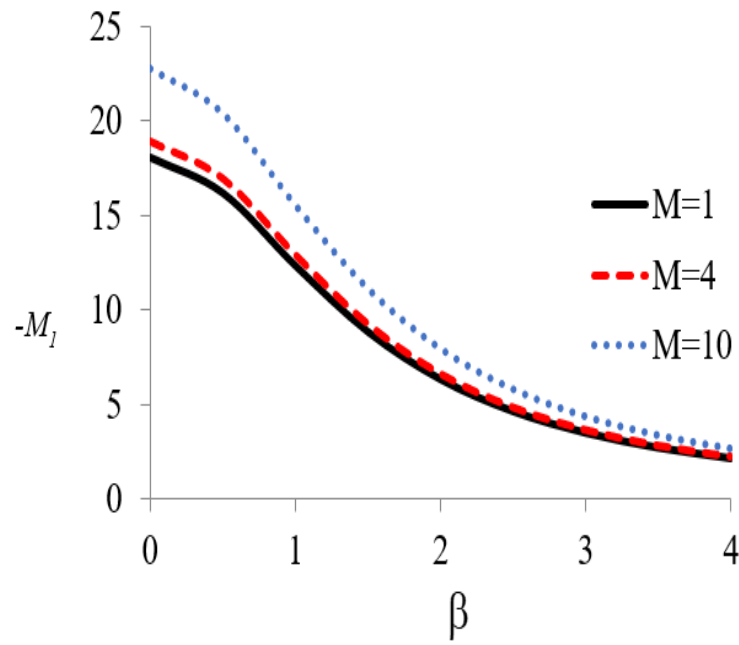

Figure 5. The plot of asymptotic dispersion coefficient vs. absorption parameter for different values of magnetic drag.

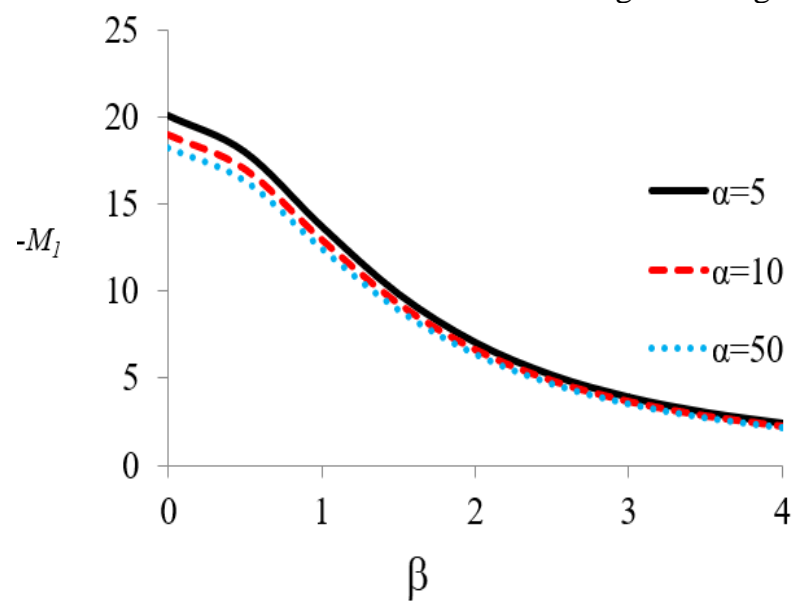

Figure 6. The plot of asymptotic dispersion coefficient vs. absorption parameter for different values of permeability.

\section{Conclusions}

This paper presents mass transfer in the presence of a catheter and magnetic field. Induced catheter creates an annual region in which mass transfer is studied using modified Gill and Sankarasubramanian model. Magnetic field influences in increasing absorption and convection. An increase in catheter radius increases the velocity. The absorption by a wall is enhanced by the presence of a magnetic field which causes more solute to get convected to the wall. Permeability increases velocity near the center increases but closer to the wall, velocity decreases. Diffusion coefficient increases with an increase in permeability due to enhancement in convection of fluid towards the wall thereby increasing absorption. Peclet number and magnetic field influence diffusion significantly. The effect of an increase in a magnetic field is to create pulsatile motion.

\section{Funding}

This research received no external funding. 


\section{Acknowledgments}

The authors would like to thank the anonymous reviewers for their valuable comments and suggestions to improve the quality of the paper.

\section{Conflicts of Interest}

The authors declare no conflict of interest.

\section{References}

1. Sarkar, A.; Jayaraman, G. The effect of wall absorption on dispersion in annular flows. Acta Mechanica 2002,158, 105-119, https://doi.org/10.1007/bf01463173.

2. Sankarasubramanian, R.; Gill, W.N. Unsteady convective diffusion with interphase mass transfer. Proceedings of the Royal Society of London. A. Mathematical and Physical Sciences 1973, 333, 115-132, https://doi.org/10.1098/rspa.1973.0051.

3. Mazumder, B.S.; Das, S.K. Effect of boundary reaction on solute dispersion in pulsatile flow through tube. Journal of Fluid Mechanics 1992, 239, 523-549, https://doi.org/10.1017/s002211209200452x.

4. Jiang,Y.; Grotberg, J.B. Bolus contaminant dispersion in oscillatory tube flow with conductive walls. Journal of Biomechanical Engineering 1993, 115, 424-431, https://doi.org/10.1115/1.2895507.

5. Balasubramanian, V.; Jayaraman, G.; Iyengar, S.R.K. Effect of secondary flows oncontaminant dispersion with weak boundary absorption. Applied Mathematical Modelling 1997, 21, 275-285, https://doi.org/10.1016/s0307-904x(97)00015-2.

6. Pedley, T.J.; Kamm, R.D. The effect of secondary motion on axial transport in oscillatory tube flow. Journal of Fluid Mechanics 1988, 193, 347-367, https://doi.org/10.1017/s0022112088002174.

7. Srivastava, V.P. Particle-fluid suspension model of blood flow through stenotic vessels with applications. International Journal of Bio-Medical Computing 1995, 38, 141-54, 1, https://doi.org/10.1016/00207101(94)01046-4.

8. Umadevi, B.; Dinesh, P.A.; Indira, R.R.; Vinay, C.V. The effect of particle drag and wall absorption on mass transfer in concentric annulus flows, Mapana Journal of Sciences 2011, 10, 1-13, https://doi.org/10.12723/mjs.18.1.

9. Ahmed M, B.; Hanan, E.; Osama, O.; Kholmirzo, T. K.; Tarek, H.; Medhat, A. I. Effect of nano metal oxides on heme molecule: molecular and biomolecular approaches. Biointerface Research in Applied Chemistry 2019, 10, 4837-4845, https://doi.org/10.33263/briac101.837845.

10. Kumar, T.S.; Kumar, B.R.; Makinde, O.D.; Kumar, A.G.V. Magneto-convective heat transfer in micropolar nanofluid over a stretching sheet with non-uniform heat source/sink. Defect and Diffusion Forum 2018, 387, 78-90, https://doi.org/10.4028/www.scientific.net/ddf.387.78.

11. Kumar T. S. Impact of Lorentz force on free convection flow of a viscous fluid past an infinite vertical plate. SN Applied Sciences 2019, 1, 1-14, https://doi.org/10.1007/s42452-019-1292-8.

12. Pedram, E.; Ehsan, K. Experimental investigation of rheological properties and formation damage of waterbased drilling fluids in the presence of $\mathrm{Al} 2 \mathrm{O} 3, \mathrm{Fe} 3 \mathrm{O} 4$, and $\mathrm{TiO} 2$ nanoparticles. Biointerface Research in Applied Chemistry 2020, 10, 5886 -5894, https://doi.org/10.33263/briac104.886894.

13. Kumar, T. S.; Dinesh, P.A.; Makinde, O.D. Impact of Lorentz force and viscous dissipation on unsteady nanofluid convection flow over an exponentially moving vertical plate. Mathematical Models and Computer Simulations 2020, 12, 631-646, https://doi.org/10.1134/s2070048220040110.

14. Shah, Z.; Alzahrani, E.O.; Dawar, A.; Ullah, A.; Khan, I. Influence of cattaneo-christov model on DarcyForchheimer flow of micropolar ferrofluid over a stretching/shrinking sheet. International Communications in Heat and Mass Transfer 2020, 110, 104385, https://doi.org/10.1016/j.icheatmasstransfer.2019.104385.

15. Kumaresan, E.; Sravan Kumar, T.; Suresh Babu, R.; MHD Slip Flow and Heat Transfer of Cu-Fe3O4/ Ethylene Glycol-Based Hybrid Nanofluid over a Stretching Surface. Biointerface Research in Applied Chemistry 2020, 11, 11956-11968, https://doi.org/10.33263/briac114.1195611968.

16. Nadjia, S.; Nadji, M.M. Formulation and characterization of oil-in-water emulsions stabilized by saponins extracted from hedera helix algeriensis using response surface method. Biointerface Research in Applied Chemistry 2020, 10, 6282- 6292, https://doi.org/10.33263/briac105.62826292. 
17. Shah, Z.; Alzahrani, E.O.; Dawar, A.; Ullah, A.; Khan, I. Influence of Cattaneo-Christov model on DarcyForchheimer flow of micropolar ferrofluid over a stretching/shrinking sheet. International Communications in Heat and Mass Transfer 2020, 110, 104385, https://doi.org/10.1016/j.icheatmasstransfer.2019.104385.

18. Ibrahim, W.; Gadisa, G. Nonlinear convective boundary layer flow of micropolar-couple stress nanofluids past permeable stretching sheet using Cattaneo-Christov heat and mass flux model. Heat Transfer 2020, 49, 2521-2550, https://doi.org/10.1002/htj.21733.

19. Naveed Khan, M.; Nadeem, S.; Muhammad, N. Micropolar fluid flow with temperature-dependent transport properties. Heat Transfer 2020, 49, 2375-2389, https://doi.org/10.1002/htj.21726.

20. Sulochana S.; Kshirabdhitanaya, D.; Malabika, T. A comparative study on the effect of temperature and concentration on density, sound velocity and their derived properties for diclofenac potassium in aqueous urea media. Biointerface Research in Applied Chemistry 2020, 10, 6377-6388, https://doi.org/10.33263/briac105.63776388.

21. Rafique, K.; Anwar, M.I.; Misiran, M.; Asjad, M.I. Energy and mass transport of micropolar nanofluid flow over an inclined surface with Keller-Box simulation. Heat Transfer 2020, 49, 4592-4611, https://doi.org/10.1002/htj.21843.

22. Khan, W.A.; Rashad, A. M.; EL-Kabeir, S.M.M.; EL-Hakiem, A.M.A. Framing the MHD micropolar nanofluid flow in natural convection heat transfer over a radiative truncated cone. Processes 2020, 8, 379, https://doi.org/10.3390/pr8040379.

23. Shobha Poovani, J.; Vidya, K.; Neeraja, G.; Sravan Kumar, T. Natural convective effects on MHD boundary layer nanofluid flow over an exponentially accelerating vertical plate. Biointerface Research in Applied Chemistry 2021, 11,13790 - 13805, https://doi.org/10.33263/BRIAC116.1379013805.

24. Agnishwar, G.; Mahashweta Mitra, G.; Pragya, P.; Seenuvasan, R.; Koyeli, G. Nano-Technology in detection of food toxins focus on the dairy products. Biointerface Research in Applied Chemistry 2021, 11, 14155 14172, https://doi.org/10.33263/BRIAC116.1415514172. 